\title{
Concealed Cause of Lumbosacral Plexopathy
}

\author{
John Jackson, Jason Webb, Ewan Bigsby \\ Department of Trauma and Orthopaedic, Soutmead Hospital, Bristol, United Kingdom
}

Cite this article as: Jackson J, Webb J, Bigsby E. Concealed Cause of Lumbosacral Plexopathy. J Emerg Med Case Rep 2017; 8: 69-72.

\begin{abstract}
Introduction: Lumbosacral plexopathies are rare in patients who present to both primary care and the accident and emergency departments. Presenting symptoms are often non-specific, such as lower back pain, sciatica, or pelvic pain, making diagnosis difficult. Furthermore, there are numerous and diverse causes of lumbosacral plexopathy.

Case Report: We present a case of a previously well 27-year-old female who presented to the accident and emergency department with hip pain, difficult walking, and altered sensation in her leg. She was referred to the orthopedic team, where magnetic resonance imaging (MRI) suggested that necrotizing fasciitis was a possible diagnosis because of abundant edema and muscle swelling and thus subsequently underwent surgical exploration. Following subsequent investigation, her symptoms and MRI features were determined to be a result of lumbosacral plexopathy secondary to immobility with rhabdomyolysis.

Conclusion: This case highlights the importance of a robust initial clinical history accompanied with appropriate investigations to elicit an accurate diagnosis and also discusses lumbosacral plexopathies in general.
\end{abstract}

Keywords: Lumbosacral, plexopathy, plexus, rhabdomyolysis

Received: 06.11.2016 Accepted: 26.12.2016 Available Online Date: 05.05.2017

\section{Introduction}

The lumbosacral plexus comprises nerve roots from the twelfth thoracic to the fourth sacral nerves. The nerves of the plexus run from the lumbosacral spine via the posterolateral pelvic walls to supply the lower back, pelvis, and lower limbs. Lumbosacral plexopathies are rare in patients who present to both primary care and the accident and emergency department. Presenting symptoms are often non-specific, such as lower back pain, sciatica, or pelvic pain, making diagnosis difficult (1).

\section{Case Report}

\section{Case history}

A 27-year-old Caucasian woman presented to the accident and emergency department in September 2014 with a 24-h history of left hip pain, difficult walking, and altered sensation in her leg. She was referred to the trauma and orthopedic teams. Pain was present in the groin and buttock, radiating down to the knee. There was no history of trauma. She had a past medical history of depression for which she took pregabalin and quetiapine, which was started 6 weeks before. She had no known drug allergies and lived alone.

On examination, she was afebrile and hemodynamically stable. There was maximal tenderness over her left ischial tuberosity, with minor tenderness in the buttock but not in the groin. Passive hip movements were pain free. The abdomen was soft and non-tender. There was a graze on the extensor surface of her left elbow, with associated minor erythema but no tracking cel-

This study was presented at the $12^{\text {th }}$ International Pediatric Emergency Medicine and Intensive Care Congress and 8th Pediatric Emergency Medicine and Intensive Care Nursing Congress, 16-19 December 2015, Antalya, Turkey. 
lulitis. Neurological assessment revealed reduced sensation in the left $L 1-L 3$ dermatomes and a four of five power on Medical Research Council grading of hip flexion, knee extension, and ankle dorsiflexion. Reflexes at the knee and ankle were brisk compared with those at the contralateral side, and ankle clonus was present with seven beats. Examination of the right lower limb revealed reduced sensation in the $L 4$ dermatome but full power and normal reflexes with no ankle clonus. Digital rectal examination was normal, with normal perianal sensation. Pedal pulses were present bilaterally. A postmicturition bladder scan revealed $>700 \mathrm{~mL}$ of residual urine, and on passing a catheter, a residual urine of $>1000 \mathrm{~mL}$ was obtained. Catheter tug sensation was also present.

\section{Investigations}

Laboratory test results on admission revealed leukocytosis of 15000/ $\mathrm{mm}^{3}$ (normal range, $4500-10000 / \mathrm{mm}^{3}$ ) and an elevated C-reactive protein (CRP) level of $190 \mathrm{mg} / \mathrm{L}$ (normal range, <5 mg/L). Lumbar, pelvic, and hip radiographs were normal. Given the abnormal neurological examination, urgent magnetic resonance imaging (MRI) of the lumbar spine and pelvis was performed. MRI demonstrated extensive edema extending through the left sciatic foramen and that involved the lumbosacral plexus and swelling of multiple muscle and fascial groups, with particular involvement of the left gluteus musculature (Figure 1).

Because of the extensive edema, a musculoskeletal radiologist believed that necrotizing fasciitis should be among the differential diagnoses. The patient was reviewed by the plastic surgery and general surgery teams and taken to the operating theater. Intra-operative findings revealed significant edema of the proximal left buttock muscles but with no features of necrotizing fasciitis. Muscle biopsies were obtained for microbiological and histological examinations as myositis of unknown etiology was considered.

The case was discussed with a rheumatologist who took over the care of the patient and performed additional specialist blood tests, including serum creatine kinase (CK), anti-nuclear antibodies, and rheumatoid factor testing. These revealed an elevated serum CK level of $33360 \mathrm{IU} / \mathrm{L}$ (normal range, 60-174 IU/L), while the remaining blood test results were normal. At this stage, the differential diagnoses included dermatomyositis, idiopathic myositis, or myositis from a quetiapine drug reaction.

\section{Treatment}

The patient was intravenously administered a 5-day course of antibiotics on the advice of microbiologists, following the equivocal MRI report, despite the intra-operative findings. A 5-day course of prednisolone was initiated, and quetiapine was discontinued. Throughout her 7-day inpatient stay, all blood test results returned to normal, and she remained clinically stable. She was discharged with outpatient rheumatology follow-up, pending the results of her muscle biopsy.

Muscle biopsy report at 10 days revealed non-specific changes in the form of a variation in muscle fiber size with pale staining, motheaten, and vacuolated fibers. This discounted dermatomyositis or

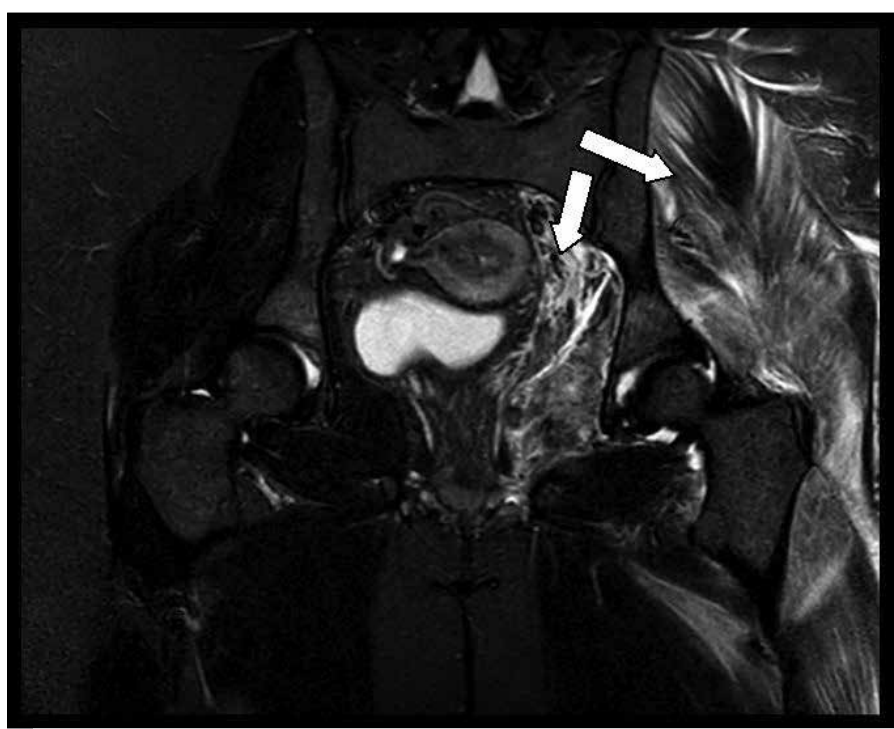

FIGURE 1. Coronal slice of MRI-short-T1 inversion recovery (STIR) sequence showing extensive edema and swelling of multiple muscles in both thighs and the pelvis, with particular involvement of the left gluteus musculature (white arrows)

myositis as a diagnosis and was more consistent with a diagnosis of rhabdomyolysis.

\section{Outcome}

Prior to her outpatient follow-up, the rheumatology team was contacted by the patient's general practitioner, who reported that the patient had disclosed that she had been stockpiling antidepressant tablets for 2 months and had taken an overdose 2 days before presenting to the accident and emergency department. She reported waking on the floor on the day of admission, and she believed that she had been unconscious for 24-48 h. In view of this additional information and combined with previous results, a diagnosis of rhabdomyolysis was made on the basis of prolonged immobility secondary to antidepressant overdose. The patient was subsequently followed up by mental health services.

Informed consent was obtained from the patient for publication of this case.

\section{Discussion}

There are numerous and diverse causes of lumbosacral plexopathy (Table 1), with non-specific computed tomography (CT) and MRI findings (1). Therefore, clinicians heavily rely on clinical history and examination findings. However, the diagnosis is further complicated by the diverse symptoms and various clinical findings depending on which part of the plexus is involved (2). The presenting features of a lumbosacral plexopathy, as with other peripheral nerve lesions, include neuropathic pain, paresthesia, allodynia, weakness, and muscle wasting. The course and prognosis of lumbosacral plexopathy is dependent on the underlying pathophysiology, especially on whether a treatable cause has been identified.

In the current case, lumbosacral plexopathy was considered to have been caused owing to extensive edema and fluid present around 
Table 1. Causes of lumbosacral plexopathy

\begin{tabular}{|c|c|}
\hline Vascular & $\begin{array}{l}\text { Proximal diabetic neuropathy / diabetic amyotrophy } \\
\text { Vasculitis in connective tissue disease (polyarteritis } \\
\text { nodosa, rheumatoid arthritis, systemic lupus } \\
\text { erythematosus) }\end{array}$ \\
\hline Trauma & $\begin{array}{l}\text { Pelvic ring disruption } \\
\text { Obstetric complications } \\
\text { Pelvic surgery }\end{array}$ \\
\hline Compressive & $\begin{array}{l}\text { Tumour; retroperitoneal or pelvic } \\
\text { Abdominal aortic or iliac aneurysm } \\
\text { Retroperitoneal haematoma or abscess } \\
\text { Rhabdomyolysis } \\
\text { Gluteal compartment syndrome }\end{array}$ \\
\hline Idiopathic & $\begin{array}{l}\text { Lumbosacral plexus neuropathy } \\
\text { Neuralgic amyotrophy }\end{array}$ \\
\hline Hereditary & $\begin{array}{l}\text { Hereditary neuralgic amyotrophy (HNA) } \\
\text { Hereditary neuropathy with liability to pressure } \\
\text { palsies (HNPP) }\end{array}$ \\
\hline Infectious & $\begin{array}{l}\text { Herpes simplex (type II) or Herpes zoster } \\
\text { Borrelia } \\
\text { Mycobacterium tuberculosis }\end{array}$ \\
\hline latrogenic & $\begin{array}{l}\text { Radiotherapy induced } \\
\text { Femoral vein catheterisation }\end{array}$ \\
\hline
\end{tabular}

the plexus, which was a consequence of rhabdomyolysis as a result of sustained pressure that occurred because of immobility caused by drug overdose. While the prevalence of depression in the general population is believed to be $2 \%-3 \%$, it can be as high as $22 \%-32 \%$ in patients who present to the accident and emergency department $(3,4)$. Identifying depression is important as it can contribute to the patient's presenting condition and can have a profound effect on their outcomes. Drug overdose can result in immobility, and if patients are not promptly found, it can lead to pressure sores, compressive neuropathies, and in rare cases, rhabdomyolysis $(5,6)$. Rhabdomyolysis is a syndrome in which skeletal muscle disintegration results in the release of large quantities of toxic muscle cell components into the plasma. While the commonest causes of acute rhabdomyolysis were crush injuries during conflict and natural disasters, a recent study reported drugs and alcohol to be the causative agents in up to $81 \%$ of cases (5). Drug-induced rhabdomyolysis can be divided into either primary or secondary myotoxic effects. Primary toxic-induced rhabdomyolysis is caused by a direct insult on the skeletal myocyte function and integrity by the pharmacological agent. Secondary toxic effects can occur as a result of overdose with local muscle compression in coma and prolonged seizures, as well as in metabolic abnormalities. In cases associated with coma, the pathophysiology is secondary to local pressure necrosis caused by compression of the patient's own body. The local pressure results in an edema-ischemia cycle that results in compartmental tamponade, muscle breakdown, and peripheral neuropathy $(5,6)$.

The only previous accounts of lumbosacral plexopathy from rhabdomyolysis have been usually from prolonged immobility from alcohol (7, 8), although Kao et al. (9) did report on a 36-year-old woman with lum- bosacral plexopathy from a presumed gluteal compartment syndrome, which resulted from immobility caused by drug overdose. Jeon et al. (7) reported a case of a 57-year-old alcoholic who presented with gluteal pain, bilateral lower limb weakness, and reduced sensation in the $L 5$ and S1 dermatomes. They used serum markers, MRI, and Technetium-99 hydroxydiphosphonate scintigraphy to diagnose lumbosacral plexopathy secondary to rhabdomyolysis. The patient recovered full function with supportive management. In a similar case, Pramila et al. (8) reported on a 51-year-old woman with a history of alcohol excess, who slept for 12 hours after binge drinking and awoke with profound right lower limb weakness, although with no sensory disturbance. Laboratory test results revealed serum parameters to be consistent with rhabdomyolysis (CK, $55000 \mathrm{IU} / \mathrm{L}$ ), and pelvic MRI demonstrated soft tissue swelling that involved the right lumbosacral plexus. The patient walked independently on discharge using a right foot orthosis; after 8 weeks of outpatient physiotherapy, she achieved good recovery of the right lower limb. Finally, Kao et al. (9) reported a case of a 36-year-old woman who developed a lumbosacral plexus injury with suspected gluteal compartment syndrome after a sedative drug overdose with prolonged immobilization of approximately $12 \mathrm{~h}$. The patient presented with right lower limb weakness and ipsilateral thigh and buttock pain; serum laboratory results were consistent with rhabdomyolysis (CK, $1173 \mathrm{IU} / \mathrm{L})$. CT revealed marked soft tissue swelling of the right gluteal muscles with diffuse subcutaneous lymphedema. Compartment pressures were not measured, but the patient was conservatively managed. At a 2-year follow-up, the patient was able to walk independently, but the foot drop did not recover.

\section{Learning points}

- Clinicians should consider lumbosacral plexopathy in patients with signs localized to the peripheral nervous system, which corresponded to the distribution of neither particular nerve roots nor peripheral nerves

- A robust drug and social history is of particular relevance when considering lumbosacral plexopathy

- Appreciation of depression as a significant contributor to the clinical presentation

- Consider an early MRI to identify edema

- Tissue sampling can be helpful in cases of diagnostic uncertainty

\section{Conclusion}

Lumbosacral plexopathy is a rare condition in patients who present to either primary care or tertiary centers. It is vital to take a careful history to identify evidence of immobility (secondary to drug overdose or alcohol excess). Investigations should include blood tests for infection (white blood cell count and CRP level) and for muscle breakdown (CK level) and MRI to identify muscle edema or compressive lesions. It is important to identify the underlying cause to enable treatment to be tailored to it.

Informed Consent: Informed consent for publication was obtained from the patient.

Peer-review: Externally peer-reviewed.

Author contributions: Concept - J.J., J.W., E.B.; Design - J.J., J.W., E.B.; Literature Search - J.J., J.W., E.B.;,; Writing - J.J., J.W., E.B.; Critical Reviews - J.J., J.W., E.B. 
Conflict of Interest: No conflict of interest was declared by the authors.

Financial Disclosure: The authors declared that this study has received no financial support.

\section{References}

1. Planner A, Donaghy M, Moore N. Causes of lumbosacral plexopathy. Clin Radiol 2006; 61: 987-95. [CrossRef]

2. Dyck J, Thaisetthawatkul P. Lumbosacral Plexopathy. Continuum (Minneap Minn) 2014; 20: 1343-58. [CrossRef]

3. Thomas C, Morris S. Cost of depression among adults in England in 2000. Br J Psychiatry 2003; 183: 514-9. [CrossRef]

4. Khav N, Weiland T, Jelinek G, Knott JC, Salzberg M. Depression symptoms and risk factors in adult emergency department patients: a multi-site cross-sectional prevalence survey. ISRN Emerg Med 2013; 8 .
5. Prendergast BD, George CF. Drug-induced rhabdomyolysis-mechanisms and management. Postgrad Med J 1993; 69: 333-6. [CrossRef]

6. Chaikin $\mathrm{H}$. Rhabdomyolysis secondary to drug overdose and prolonged coma. South Med J 1980; 73: 990-4. [CrossRef]

7. Jeon $\mathrm{H}, \mathrm{Cho} B, \mathrm{Oh} \mathrm{S}$, Park S. Lumbosacral plexopathy, complicating rhabdomyolysis in a 57-year-old man, presented with sudden weakness in both legs. J Korean Neurosurg Soc 2007; 42: 481-3. [CrossRef]

8. Pramila K, Kolisetty M, Brown R, Polepalle SK, Abdelmassieh NH, Henderson M. Acute Lumbosacral Plexopathy Caused by Rhabdomyolysis: A Case Report. Poster presented at The 65th Annual Assembly of the American Academy of Physical Medicine and Rehabilitation, Phoenix, Az 2004. Abstract retrieved from: http://www.archives-pmr.org/article/ S0003-9993\%2804\%2900858-5/abstract

9. Kao C, Yuan C, Cheng Y, Chan RC. Lumbosacral Plexus Injury and Brachial Plexus Injury Following Prolonged Compression. J Chine Med Assoc 2006; 69: 543-8.[CrossRef] 